\title{
Disulfiram deregulates HIF- $\alpha$ subunits and blunts tumor adaptation to hypoxia in hepatoma cells
}

\author{
Hye-joon PARK ${ }^{2}$, Min-sung $\mathrm{KIM}^{3}$, Kumsun $\mathrm{CHO}^{1}$, Jang-hyuk $\mathrm{YUN}^{1}$, Yong-joon $\mathrm{CHOI}^{1}$, Chung-hyun $\mathrm{CHO}^{1, *}$ \\ ${ }^{1}$ Department of Pharmacology and Biomedical Science, Seoul National University College of Medicine, 103 Daehak-ro, Jongno-gu, \\ Seoul 110-799, Korea; ${ }^{2}$ Sehwa Girls' High School, 56-7 Sinbanpo-ro, Seocho-gu, Seoul 137-040, Korea; ${ }^{3}$ Hansung Science High \\ School, 279-79 Tongil-ro, Seodaemun-gu, Seoul 120-080, Korea
}

\begin{abstract}
Aim: Disulfiram is an aldehyde dehydrogenase inhibitor that was used to treat alcoholism and showed anticancer activity, but its anticancer mechanism remains unclear. The aim of this study was to investigate the effects of disulfiram on the hypoxia-inducible factor (HIF)-driven tumor adaptation to hypoxia in vitro.

Methods: Hep3B, Huh7 and HepG2 hepatoma cells were incubated under normoxic $\left(20 \% \mathrm{O}_{2}\right)$ or hypoxic $\left(1 \% \mathrm{O}_{2}\right)$ conditions for $16 \mathrm{~h}$. The expression and activity of HIF-1 $\alpha$ and HIF-2 $\alpha$ proteins were evaluated using immunoblotting and luciferase reporter assay, respectively. Semi-quantitative RT-PCR was used to analyze HIF-mediated gene expression. Endothelial tubule formation assay was used to evaluate the anti-angiogenic effect.

Results: Hypoxia caused marked expression of HIF-1 $\alpha$ and HIF-1 $\alpha$ in the 3 hepatoma cell lines, dramatically increased HIF activity and induced the expression of HIF downstream genes (EPO, CA9, VEGF-A and PDK1) in Hep3B cells. HIF-2 $\alpha$ expression was positively correlated with the induction of hypoxic genes (CA9, VEGF-A and PDK1). Moreover, hypoxia markedly increased VEGF production and angiogenic potential of Hep3B cells. Disulfiram ( 0.3 to $2 \mu \mathrm{mol} / \mathrm{L})$ inhibited hypoxia-induced gene expression and HIF activity in a dosedependent manner. Disulfiram more effectively suppressed the viability of Hep3B cells under hypoxia, but it did not affect the cell cycle. Overexpression of HIF-2 $\alpha$ in Hep3B cells reversed the inhibitory effects of disulfiram on hypoxia-induced gene expression and cell survival under hypoxia.

Conclusion: Disulfiram deregulates the HIF-mediated hypoxic signaling pathway in hepatoma cells, which may contribute to its anticancer effect. Thus, disulfiram could be used to treat solid tumors that grow in a HIF-dependent manner.
\end{abstract}

Keywords: disulfiram; hepatoma; hypoxia; HIF-2; VEGF; angiogenesis

Acta Pharmacologica Sinica (2013) 34: 1208-1216; doi: 10.1038/aps.2013.52; published online 15 Jul 2013

\section{Introduction}

Mammalian cells have versatile adaptive mechanisms to survive in oxygen-deficient (hypoxic) conditions ${ }^{[1]}$. These adaptations require the expression of numerous genes that are commonly regulated by the HIF (hypoxia-inducible factor) transcription factors HIF- $\alpha$ and HIF- $\beta$ (alternatively named ARNT $)^{[2]}$. Of the HIF- $\alpha$ isoforms, HIF-1 $\alpha$ and HIF-2 $\alpha$ play distinct roles in determining cell fate by regulating gene expression in different ways. ARNT is a common partner for HIF-1/2 $\alpha$ and functions to help HIF- $\alpha$ bind to DNA ${ }^{[3]}$. HIFmediated adaptation to hypoxia is essential for organisms to cope with accidental hypoxia. However, HIF can be a lifethreatening factor in cancer patients because cancers also

\footnotetext{
* To whom correspondence should be addressed.

E-mail iamhyun@snu.ac.kr

Received 2012-12-05 Accepted 2013-04-08
}

adopt HIF to survive under hypoxia and to expand their territories beyond their original sites. For this reason, HIF is considered an emerging target for cancer therapy ${ }^{[4]}$.

Disulfiram, an aldehyde dehydrogenase inhibitor, was approved by the US FDA in 1951 as a therapy for alcoholism. It induces the accumulation of acetaldehyde produced from ingested alcohol, resulting in unendurable hangover symptoms, the so-called "disulfiram-ethanol reaction" ${ }^{[5]}$. Disulfiram is a symmetric compound with a $S=C-S-S-C=S$ functional group (Figure 1A), which disables aldehyde dehydrogenase by forming a disulfide bond with the enzyme. Interestingly, this moiety also gives disulfiram the ability to bind to various organic or inorganic molecules ${ }^{[6-8]}$. Given its thiol-reactive binding properties, disulfiram is thought to interact with various types of proteins. If tumor-promoting proteins are targeted by disulfiram, then disulfiram might have anticancer activities. Indeed, an article entitled "Disulfiram and tumor 
inhibition" was first recorded on PubMed in $1966^{[9]}$, and many reports regarding the anticancer effect of disulfiram have been published since then. The anticancer properties of disulfiram have been shown to affect carcinogenic activation ${ }^{[10]}, \mathrm{Cu} / \mathrm{Zn}$ $\mathrm{SOD}^{[11]}, \mathrm{NF}-\mathrm{KB}^{[12]}$, matrix metalloproteinases ${ }^{[13]}$, phosphoinositide 3-kinase ${ }^{[14]}$, P-glycoprotein ${ }^{[15]}$, proteasomal enzymes ${ }^{[16]}$, and DNA methyltransferases ${ }^{[17]}$. However, the detailed molecular mechanism underlying its anticancer action remains unclear.

According to the previous literature, disulfiram appears to inhibit tumor growth via multiple pathways. Nonetheless, few studies have investigated the effect of disulfiram on HIF-mediated tumor progression. In the present study, we addressed this research point and found that disulfiram inhibits the hypoxic response in hepatoma cells by deregulating the HIF signaling pathway. To the best of our knowledge, this is the report suggesting the development of disulfiram as an agent for treating HIF-related diseases including malignant tumors.

\section{Materials and methods Reagents}

Disulfiram and other chemicals were purchased from SigmaAldrich Corp (St Louis, MO, USA), and $\left[\mathrm{a}^{-32} \mathrm{P}\right] \mathrm{CTP}$ was from NEN Life Science (Boston, MA, USA). Culture media and fetal calf serum (FBS) were purchased from GIBCO/BRL (Grand Island, NY, USA). Anti-HIF-1a antiserum was kindly provided by Dr Jong-wan PARK (Seoul National University, Korea), and anti-HIF-2a was obtained from Novus Biologicals (Littleton, CO, USA). Other primary and HRP-conjugated secondary antibodies were purchased from Santa Cruz Biotechnology (Santa Cruz, CA, USA).

\section{Cell lines and culture}

Hep3B, Huh7, and HepG2 hepatoma cells were cultured in a-modified Eagle's medium or in Dulbecco's modified Eagle's medium, which was supplemented with $10 \%$ heat-inactivated FBS, 100 units/mL penicillin, and $100 \mu \mathrm{g} / \mathrm{mL}$ streptomycin. All cells were grown in a humidified $5 \% \mathrm{CO}_{2}$ atmosphere at $37^{\circ} \mathrm{C}$ in an incubator, in which oxygen tension was held at either $140 \mathrm{mmHg}\left(20 \% \mathrm{O}_{2}, v / v\right.$, normoxic conditions $)$ or 7 $\mathrm{mmHg}\left(1 \% \mathrm{O}_{2}, v / v\right.$, hypoxic conditions). Cells were treated with disulfiram for one hour before hypoxic incubation.

\section{Plasmids and transfection}

The plasmid containing HIF-2a was a kind gift from Dr Jongwan PARK (Seoul National University, Korea). The fulllength cDNA of HIF-2a was inserted into the pcDNA vector. For transient protein expression, cells at approximately $40 \%$ confluence were transfected with the plasmid using Lipofectamine (Invitrogen, Carlsbad, CA, USA). Cells were allowed to stabilize for $48 \mathrm{~h}$ before being assayed.

\section{Cell viability assays}

To determine where there was any acute toxicity associated with disulfiram, Hep3B cells were treated with disulfiram for
$16 \mathrm{~h}$ and then incubated with an MTT labeling reagent (SigmaAldrich, St Louis, MI, USA) in a $\mathrm{CO}_{2}$ chamber for $3 \mathrm{~h}$. Blue formazan crystals were solubilized with acidified isopropanol, and formazan levels were determined at $570 \mathrm{~nm}$. To identify any long-term effects of disulfiram on cell survival, we counted viable cells using a trypan blue exclusion assay. After treatment with disulfiram for $48 \mathrm{~h}$, Hep3B cells were detached in a trypsin-EDTA solution, and the cell suspension was mixed with $0.4 \%$ trypan blue solution $(1: 1, v: v)$. Unstained, viable cells were counted on a hemocytometer.

\section{Reporter assay}

Luciferase reporter genes containing the hypoxia response element (HRE) of the EPO gene were generously donated by Dr Eric HUANG (University of Utah, USA). Hep3B cells were co-transfected with $0.5 \mu \mathrm{g}$ of the reporter plasmid and cytomegalovirus- $\beta$-gal plasmids using the calcium phosphate method. pcDNA was added to ensure that the final DNA concentrations in the control and experimental groups were at similar levels. After stabilization for $48 \mathrm{~h}$, cells were incubated under normoxic or hypoxic conditions for $16 \mathrm{~h}$. Cells were then lysed to determine luciferase and $\beta$-gal activities. Luciferase activities were analyzed using a Lumat LB9507 luminometer (Berthold Technologies, Bad Wildbad, Germany), and $\beta$-gal assays were performed to normalize transfection efficiencies.

\section{Semi-quantitative RT-PCR analysis}

To quantify mRNA levels, highly sensitive RT-PCR was performed $^{[18]}$. RNA was isolated with TRIzol Reagent (Invitrogen) and quantified by measuring the absorbance at $260 \mathrm{~nm}$. One microgram of RNA was used for reverse transcription using the Superscript One-Step kit (Invitrogen), and the cDNA was amplified over 25 PCR cycles with $\left[\mathrm{a}^{32} \mathrm{P}\right] \mathrm{dCTP}$. Five microliters of the PCR products was electrophoresed on a $4 \%$ polyacrylamide gel at $100 \mathrm{~V}$ in TAE buffer, and the dried gels were autoradiographed. The sequences of primers are summarized in Table 1.

\section{Immunoblotting}

Total proteins were separated on an $8 \%$ SDS/polyacrylamide gel and transferred to Immobilon-P membranes (Millipore; Bedford, MA, USA). Membranes were then blocked with 5\% nonfat milk at room temperature for $1 \mathrm{~h}$ and incubated overnight at $4{ }^{\circ} \mathrm{C}$ with primary antibodies (1:5000). Horseradish peroxidase-conjugated anti-rabbit antiserum was used as a secondary antibody (1:5000), and antigen-antibody complexes were visualized using an Enhanced Chemiluminescence Plus kit (Amersham Biosciences, Piscataway, NJ, USA). To quantify protein levels, the protein bands were scanned, and their intensities and areas were analyzed using ImageJ (NIH, USA). Each protein level was estimated by multiplying its intensity and area, and the results (the mean of three experiments) are presented as the relative (\%) values to the hypoxic, untreated group (100\%). 
Table 1. Nucleotide sequences of primers used in experiments.

\begin{tabular}{lll}
\hline Targets of PCR & Forward primers $\left(5^{\prime}\right.$ to $\left.3^{\prime}\right)$ & Reverse primers $\left(5^{\prime}\right.$ to $\left.3^{\prime}\right)$ \\
\hline Human EPO & AACAATCACTGCTGACACTT & AGAGTTGCTCTCTGGACAGT \\
Human CA9 & TATGAGGGGTCTCTGACTACA & TTCTCATCTGCACAAGGAAC \\
Human VEGF-A & AACTTTCTGCTGTCTTGG & TTTGGTCTGCATTCACAT \\
Human PDK1 & GAAGCATAAATCCAAACTGC & AACCCTCTAGGGAATACAGC \\
Human $\beta$-actin & AAGAGAGGCATCCTCACCCT & ATCTCTTGCTCGAAGTCCAG \\
\hline
\end{tabular}

\section{Endothelial tube formation assay}

Human umbilical vein endothelial cells (HUVECs) were detached and resuspended in conditioned medium from Hep3B cells that had been incubated under normoxia or hypoxia for $48 \mathrm{~h}$. Fifty microliters of cell suspension $\left(5 \times 10^{4}\right.$ cells per well) was added to each well of a 24 -well plate that had been coated with growth factor-reduced Matrigel (BD Biosciences; San Jose, CA, USA). The HUVEC culture plate was incubated for $16 \mathrm{~h}$ at $37^{\circ} \mathrm{C}$ in a $5 \% \mathrm{CO}_{2}$ atmosphere. The plate was examined under a light microscope, and phase contrast images were captured using a digital camera. Vessel length and tube area were calculated using ImageJ.

\section{Cell cycle analysis}

Hep3B cells were cultured at $\sim 40 \%$ confluence and incubated under normoxia or hypoxia with vehicle or disulfiram for $48 \mathrm{~h}$. Cells were harvested and fixed in $75 \%$ ethanol for $30 \mathrm{~min}$ on ice. After washing with PBS, cells were labeled with propidium iodide $(0.05 \mathrm{mg} / \mathrm{mL})$ in the presence of RNase A (0.5 $\mathrm{mg} / \mathrm{mL}$ ) and incubated at room temperature in the dark for 30 min. DNA contents were analyzed using a flow cytometer. Propidium iodide incorporated into DNA was excited at 488 $\mathrm{nm}$ and detected at $650 \mathrm{~nm}$.

\section{Statistical analysis}

All data were analyzed using Microsoft Excel 2007, and the results are expressed as the mean \pm SD. The two-sided MannWhitney $U$ test was used to compare luciferase activities, cell numbers, VEGF concentrations, and angiogenesis parameters. Differences were considered significant when $P$ values were less than 0.05 .

\section{Results}

\section{Effects of disulfiram on HIF-1 $\alpha$ and HIF- $2 \alpha$ expression}

To evaluate changes in gene expression in response to hypoxia, a 16-h incubation is usually performed. Therefore, we examined whether disulfiram was toxic to Hep3B cells under such conditions. Disulfiram showed no substantial toxicity at concentrations lower than $2 \mu \mathrm{mol} / \mathrm{L}$ (Figure 1B), and thus, we treated Hep3B cells with $<2 \mu \mathrm{mol} / \mathrm{L}$ of disulfiram during the next experiments. We first checked whether disulfiram affected the expression of the HIF-1a or HIF-2a proteins. Interestingly, disulfiram reduced HIF-2a expression in a dosedependent manner, whereas it marginally increased HIF-1a in Hep3B cells (Figure 1C). Likewise, disulfiram also noticeably downregulated HIF-2a in Huh7 and HepG2 hepatoma cells (Figure 1D). Unexpectedly, HIF-1a was reduced by $2 \mu \mathrm{mol} / \mathrm{L}$ disulfiram in Huh7 and HepG2 cells, suggesting that HIF-1a regulation by disulfiram is variable among hepatoma cell lines. To measure the normoxic levels of HIF-1a and HIF-2a, we exposed immunoblots to $\mathrm{X}$-ray films for a longer period of time (10 min). Even under normoxia, HIF-2a was faintly detected, and its level was also reduced by disulfiram (Figure $1 \mathrm{E})$. In contrast, HIF-1a protein could not be detected in normoxic cells by Western blotting. Taken together, disulfiram showed a consistent suppression of HIF-2a in hepatoma cells.

\section{Functional repression of HIF by disulfiram}

HIF-1a and HIF-2a dimerize with ARNT to form the transcriptional complexes HIF-1 and HIF-2, respectively. HIF-1 and HIF-2 are present in hypoxic hepatoma cells, and both contribute to the expression of hypoxia-induced genes. As HIF-1a and HIF-2a were differentially regulated by disulfiram, it was unexpected how the net activity of HIF is affected by disulfiram. To address this question, we used a luciferase reporter system that is activated by both HIFs. When Hep3B cells were exposed to hypoxia for $16 \mathrm{~h}$, the reporter activity was markedly stimulated, and hypoxic activation was significantly attenuated by disulfiram at concentrations over 0.3 $\mu \mathrm{mol} / \mathrm{L}$ (Figure 2A). This result strongly indicates that HIF activity was repressed overall by disulfiram. To evaluate HIF-mediated changes in gene expression, we analyzed the mRNA of representative HIF downstream genes using highly sensitive RT-PCR. We found that erythropoietin (EPO), carbonic anhydrase 9 (CA9), vascular endothelial growth factor A (VEGF-A), and pyruvate dehydrogenase kinase 1 (PDK1) mRNAs increased in response to hypoxia, and all of these genes were downregulated by disulfiram in a dose-dependent manner (Figure 2B). Densitometry analyses showed that all HIF downstream mRNAs were substantially reduced by disulfiram. The induction of EPO and CA9 mRNA by hypoxia was especially sensitive to disulfiram attenuation (Figure 2C). We next examined whether disulfiram repressed the expression of these genes in Huh7 and HepG2 cells. As expected, the levels of EPO and CA9 mRNA were also highly induced in these cells during hypoxia, and these hypoxic inductions were attenuated by disulfiram (Figure 2D). These results strongly indicate that disulfiram effectively repressed the HIF signaling pathway in hepatoma cells. 
A

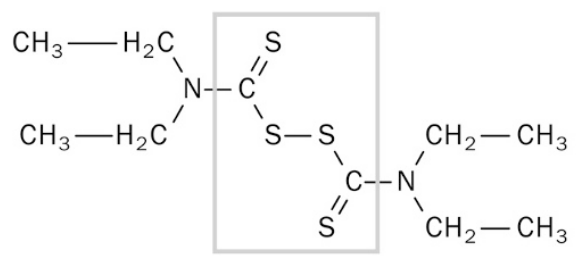

B

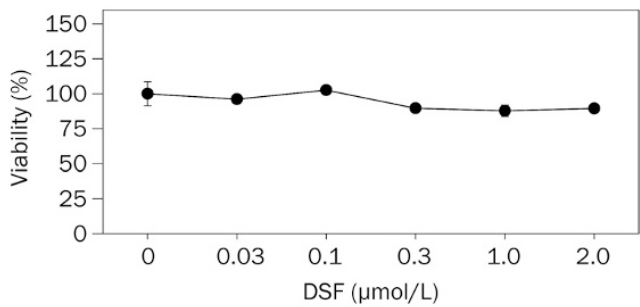

C

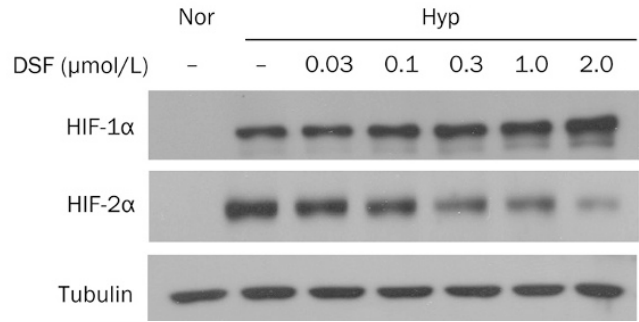

D

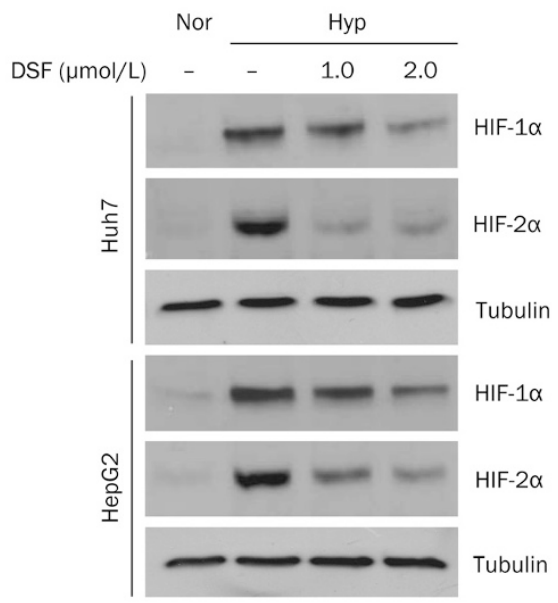

E

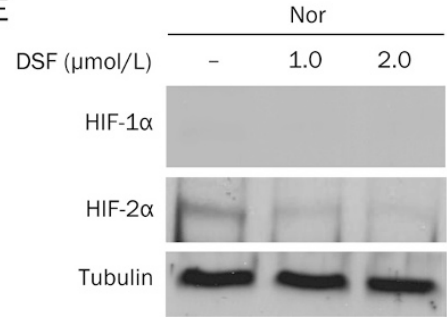

Figure 1. Effects of disulfiram on the expression of HIF-1 $\alpha$ and HIF-2 $\alpha$ under hypoxic conditions. (A) Structure of disulfiram (DSF). The core structure within the box is able to conjugate with thiol groups in proteins. (B) Hep3B cells were treated with the indicated concentrations of disulfiram for $16 \mathrm{~h}$. Cell viability was assessed by MTT staining. (C) Hep3B cells were incubated with various concentrations of disulfiram in normoxic (21\% oxygen) or hypoxic (1\% oxygen) conditions for $16 \mathrm{~h}$. Cells were lysed and immunoblotted for the indicated proteins with their specific antibodies. The immunoblots were stained with the ECL-plus kit and exposed to X-ray films for 30-60 s. (D) Huh7 and HepG2 cells were treated with disulfiram in hypoxic conditions for $16 \mathrm{~h}$, and the indicated proteins were analyzed by immunoblotting. (E) Hep3B cells were treated with disulfiram in normoxic conditions for $16 \mathrm{~h}$. Immunoblots of HIF-1/2 $\alpha$ were exposed to X-ray films for 10 min to visualize faint protein bands.

\section{Role of HIF-2 $\alpha$ in hypoxic gene expression}

At the beginning of this study, we paradoxically found that HIF-1 $a$ and HIF-2a were differentially regulated by disulfiram. However, the subsequent studies suggested that HIF-2a, rather than HIF-1a, affects the hypoxic signaling pathway in response to disulfiram. To support this hypothesis, we analyzed the relationship between HIF-2a levels and hypoxic gene levels (Figure 2E). The expression of CA9, VEGF-A, and PDK1 highly correlated with HIF-2a expression. Although EPO expression increased with HIF-2a expression, their correlation coefficient was lower than those of other genes with HIF-2a. In addition to being regulated by HIF-2a, the EPO gene may also be regulated by other factor(s) whose activity is affected by disulfiram. To understand the involvement of HIF-2 $\alpha$ in disulfiram-induced repression of hypoxia-induced genes, we overexpressed HIF-2 $\alpha$ in Hep3B cells and then examined the expression of EPO and CA9 in the presence of disulfiram. Despite HIF-2a overexpression, the mRNA levels of EPO and CA9 under hypoxia were not significantly enhanced, which suggests that endogenous HIF-2a expressed under hypoxia was sufficient for gene expression. Interest- ingly, HIF-2a overexpression substantially rescued the EPO and CA9 expression repressed by disulfiram (Figure 2F). These results support our hypothesis that disulfiram blunts the induction of hypoxia genes by suppressing HIF-2a.

\section{Effect of disulfiram on cell survival under hypoxia}

Because disulfiram deregulated the HIF-2 signaling pathway, we next addressed the effects of disulfiram on cell survival and angiogenesis under hypoxia. When Hep3B cells were treated with disulfiram for $48 \mathrm{~h}$, viable cells were reduced under normoxia in a dose-dependent manner. Moreover, disulfiram even more effectively reduced cell viability under hypoxia (Figure 3A). We then examined whether disulfiram affected the cell cycle and found that the populations of cells in each phase of the cell cycle were not changed by disulfiram under either normoxic or hypoxic conditions (Figure 3B and 3C). Disulfiram appeared to inhibit cell survival under hypoxia irrespective of the cell cycle phase. Therefore, is HIF-2a suppression responsible for hypoxic cell death by disulfiram? To answer this question, we determined the effect of disulfiram on Hep3B cells overexpressing HIF-2a. As ectopic protein lev- 
A

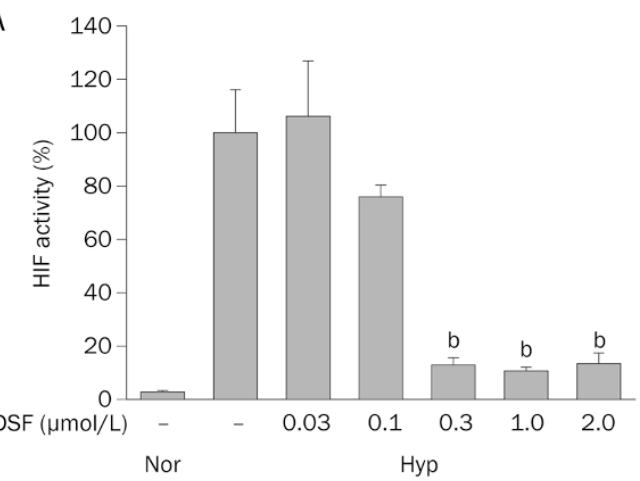

C

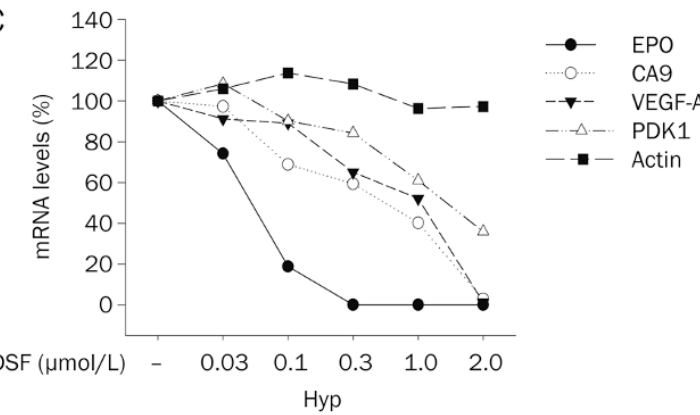

E

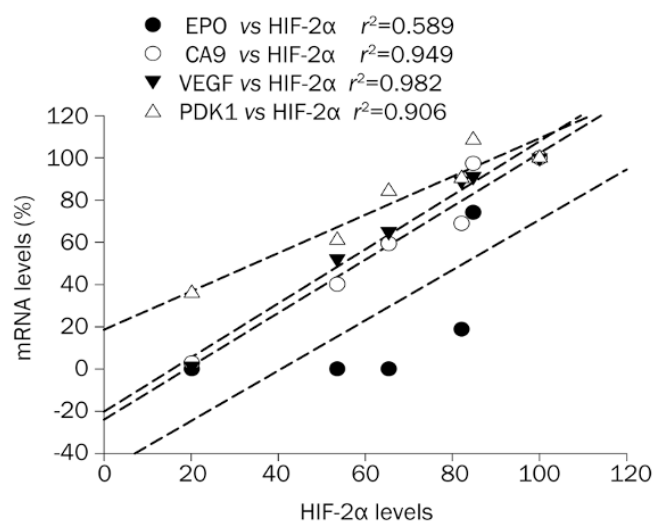

B

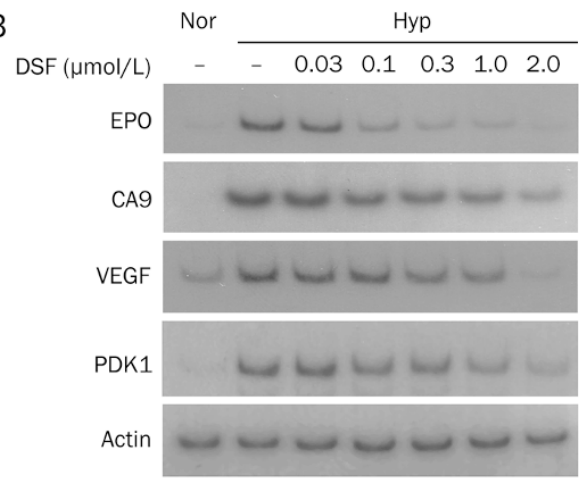

D

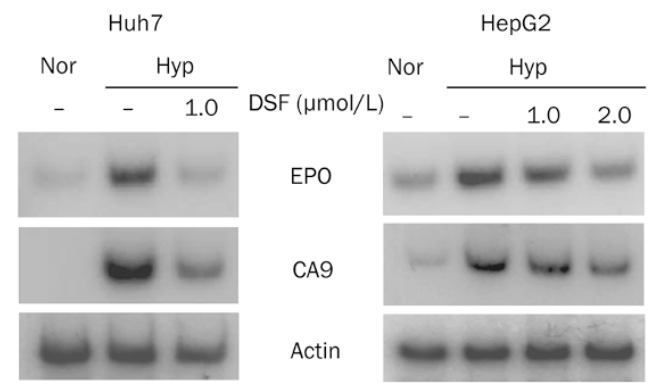

F

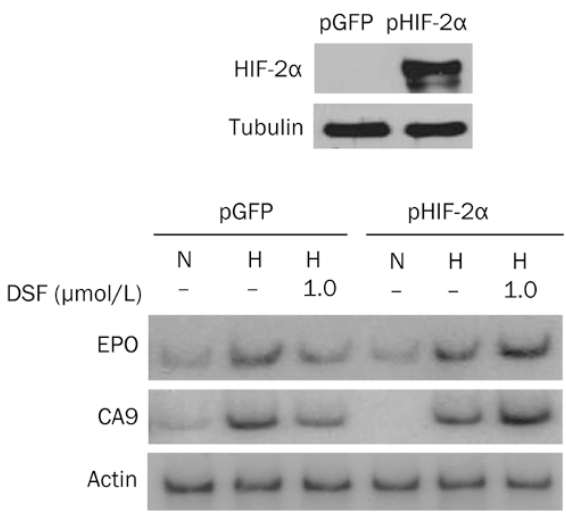

Figure 2. Effect of disulfiram on the HIF signaling pathway. (A) Hep3B cells were co-transfected with the EPO-enhancer luciferase plasmid (1 $\mu \mathrm{g}$ per 60-mm dish) and the CMV-promoter $\beta$-galactosidase plasmid (0.5 $\mu \mathrm{g})$. After stabilization for $36 \mathrm{~h}$, cells were incubated with various concentrations of disulfiram (DSF) under hypoxic conditions for $16 \mathrm{~h}$ and lysed for luciferase and $\beta$-galactosidase assays. Luciferase activity was divided by $\beta$-galactosidase activity to normalize transfection efficiency. Results are given as the relative value versus the hypoxic control and plotted as the mean \pm SD of four experiments. ${ }^{b}$ denotes the significant difference $(P<0.05)$ versus the hypoxic control value. (B) Hep3B cells were incubated with disulfiram under normoxic or hypoxic conditions for $16 \mathrm{~h}$, and total RNA was isolated with TRIzol reagent. The indicated mRNAs were amplified with $\left[\alpha-{ }^{32} \mathrm{P}\right] \mathrm{dCTP}$ by running PCR for 16-20 cycles. The radioactive PCR products were subjected to electrophoresis and autoradiography. (C) The RT-PCR bands developed on X-ray films were scanned and quantified using ImageJ. The average values from three experiments were plotted as a function of disulfiram concentration. (D) Huh7 and HepG2 cells were treated with disulfiram under hypoxic conditions for $16 \mathrm{~h}$, and the indicated mRNAs were analyzed by semi-quantitative RT-PCR. (E) The relationship between HIF-2 $\alpha$ expression and the expression of hypoxia genes. The HIF-2 $\alpha$ bands (Figure 1C) developed on X-ray films were scanned and quantified using ImageJ. The average protein levels from three experiments were plotted on the $X$-axis. The levels of hypoxia-induced mRNA presented in Figure $2 \mathrm{C}$ were plotted on the $y$-axis. The linear regression (lines) and the correlation coefficients $\left(r^{2}\right)$ were analyzed using SigmaPlot (version 2000). (F) Hep3B cells were transfected with GFP (as a control) or HIF-2 $\alpha$ plasmid (1 $\mu$ g per 100-mm dish) and stabilized for $48 \mathrm{~h}$. Cells were treated with disulfiram under hypoxic conditions for $16 \mathrm{~h}$, and the indicated mRNAs were analyzed by semi-quantitative RT-PCR. Before starting the experiment, HIF-2 $\alpha$ overexpression was verified in the transfected cells using Western blotting (upper panel).

els significantly dropped on the 4th day after transient transfection, we performed this experiment before that amount of time had passed. For this reason, after transfected cells were stabilized for $48 \mathrm{~h}$, we incubated the cells with disulfiram 
A
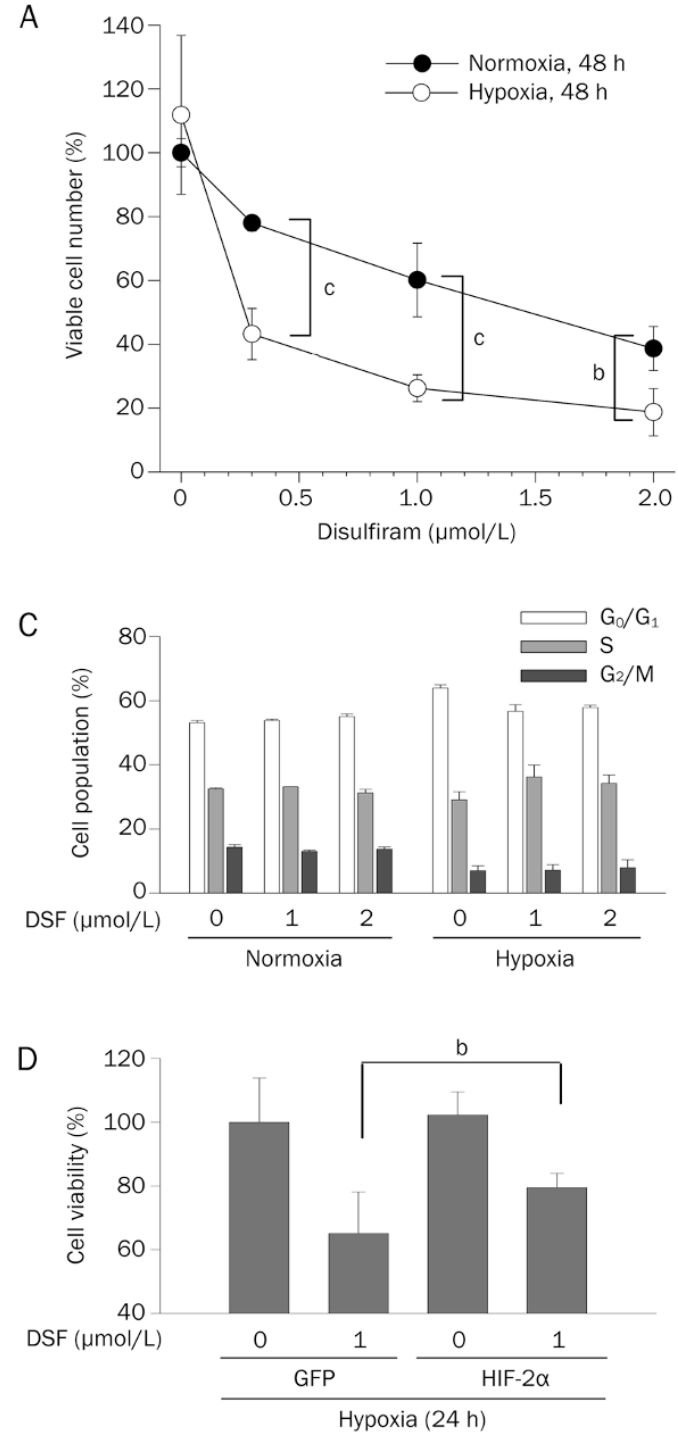

B
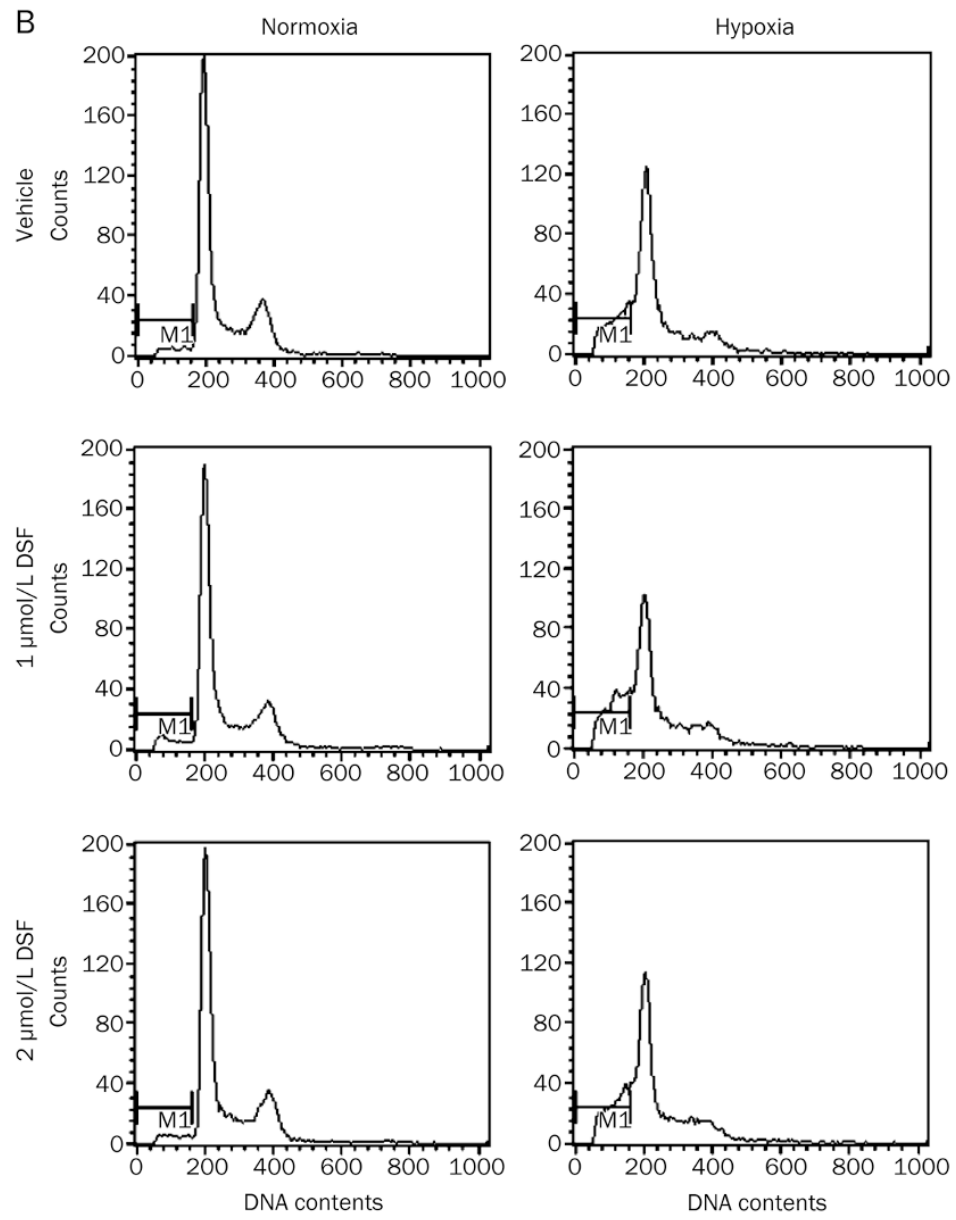

Figure 3. Effects of disulfiram on cell survival and VEGF secretion. (A) Hep3B cells were incubated with the indicated concentrations of disulfiram under normoxia or hypoxia for $48 \mathrm{~h}$. Cells were stained with trypan blue, and the unstained cells were counted as viable cells. Results are given as percentages versus the normoxic control value and plotted as the mean $\pm \mathrm{SD}$ of eight experiments. ${ }^{\mathrm{b}} P<0.05,{ }^{\mathrm{c}} P<0.01$ between the indicated groups, respectively. (B) After Hep3B cells were incubated for $48 \mathrm{~h}$ under the indicated conditions, cellular DNA was labeled with propidium iodide and analyzed by flow cytometry. (C) Flow cytometry results are given as percent proportions in $G_{0} / G_{1}, S$, and $G_{2} / M$ phases. (D) Hep3B cells were transfected with $G F P$ or HIF-2 $\alpha$ plasmid $(1 \mu \mathrm{g})$ and stabilized for $48 \mathrm{~h}$. Cells were treated with disulfiram under hypoxic conditions for $24 \mathrm{~h}$, and cell viability was assessed by MTT staining. ${ }^{b} P<0.05$ between the indicated groups.

under hypoxic conditions for $24 \mathrm{~h}$. As a consequence, under these conditions, disulfiram reduced cell viability, which was significantly recovered by HIF-2a overexpression (Figure 3D). However, the effect of HIF-2a overexpression was not sufficient to support the idea that disulfiram inhibits cell survival during hypoxia by suppressing HIF-2a. Given that the efficiency of transfection was approximately $30 \%$ in Hep3B cells, the effect of HIF-2a overexpression may be underestimated due to limited transfection efficiency in our experimental setting.

\section{Disulfiram actions against VEGF production and angiogenesis}

Because VEGF is the prime factor that induces tumor angiogenesis, we analyzed the levels of VEGF secreted from cancer cells. Hep3B cells produced VEGF at a basal level under normoxia, and VEGF production was augmented under hypoxia. In the present study, disulfiram effectively reduced VEGF production in both normoxic and hypoxic conditions (Figure 4A). To further analyze the anti-angiogenic effects of disulfiram, we performed in vitro angiogenic assays using HUVECs. When treated with conditioned media from Hep3B cells, endothelial cells connected with each other to form a web-like structure. 
A

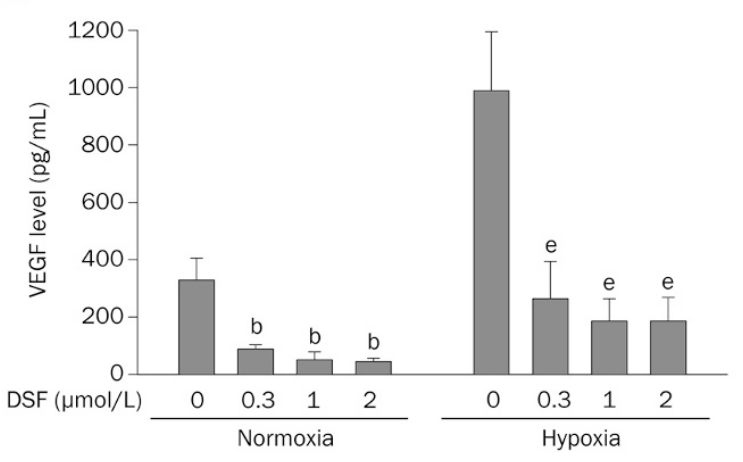

B

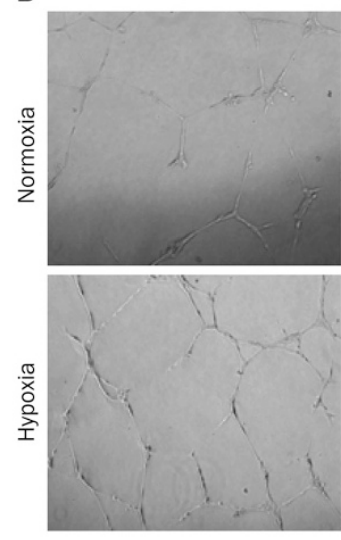

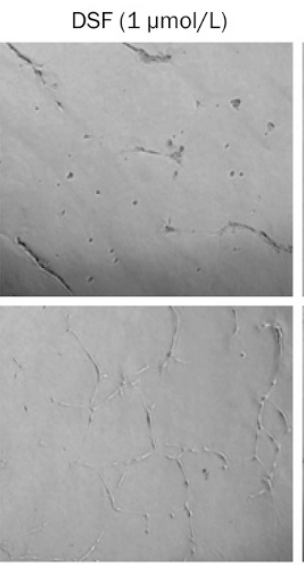

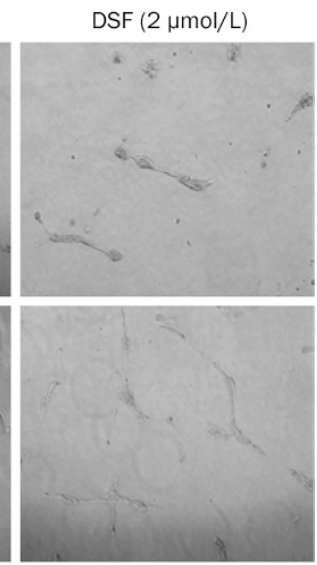

C

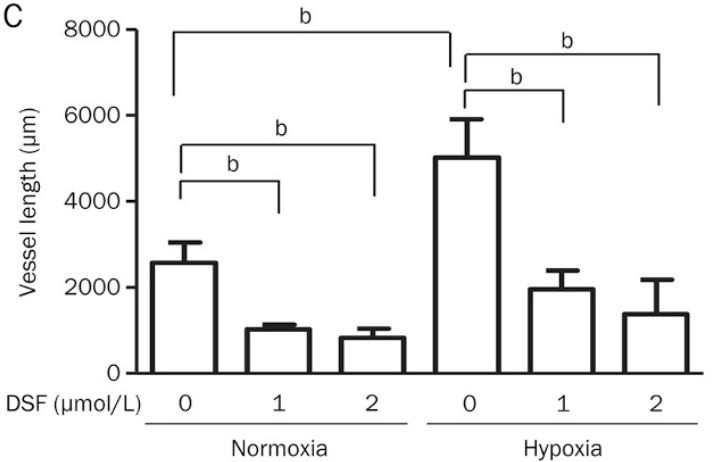

D

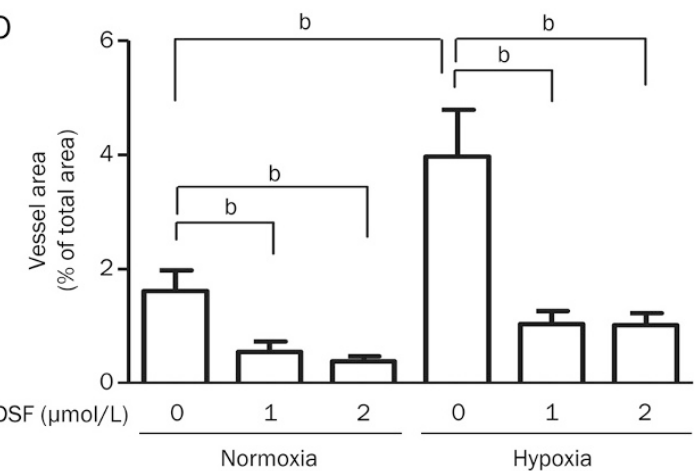

Figure 4. Effect of disulfiram on the angiogenic potential of Hep3B. (A) VEGF levels were analyzed in the culture media from Hep3B cells that had been incubated for $48 \mathrm{~h}$ under the indicated conditions using the Quantikine Human VEGF Immunoassay Kit (R\&D Systems: Minneapolis, Minnesota). The VEGF levels in the media are normalized as pg per $\mathrm{mL}$ and plotted as the mean \pm SEM of four experiments. ${ }^{\mathrm{b}} P<0.05$ vs normoxic control. ${ }^{\mathrm{e}} \mathrm{P}<0.05$ vs hypoxic control values, respectively. (B) Hep3B cells were incubated for $48 \mathrm{~h}$ under the indicated conditions to collect conditioned media. Human umbilical vein endothelial cells were cultured on Matrigel-coated plates in the conditioned media. After $16 \mathrm{~h}$, images of the cell arrangements were captured at 40×magnification under a microscope. (C, D) Vessel lengths $(\mu \mathrm{m})$ and tube areas (\% of total area) were measured using Image J and plotted as the means $\pm \mathrm{SD}$ of six experiments. ${ }^{\mathrm{b}} \mathrm{P}<0.05$ between the indicated groups.

However, this endothelial structure was incompletely formed in the media obtained from disulfiram-treated cells (Figure 4B). Image analysis showed that vessel length and tube area were both significantly reduced in endothelial cells incubated in the disulfiram-conditioned media (Figure 4C and 4D). The anti-angiogenic action of disulfiram may be attributed to the reduction in VEGF caused by disulfiram.

\section{Discussion}

Disulfiram was originally developed to help alcoholics cease drinking alcohol. However, new indications for this drug have been suggested. For example, disulfiram has been used to treat cocaine addiction ${ }^{[19]}$, metal-induced contact dermatitis $^{[20]}$, uveitis ${ }^{[21]}$, and malignant tumors ${ }^{[9-17]}$. In the present study, we found that disulfiram blunted tumor adaptation to hypoxia by deregulating the HIF-2 signaling pathway. This pharmacological action of disulfiram may at least in part underlie its anticancer effect.

It was previously believed that HIF-1 $\alpha$ and HIF-2a promote tumor progression similarly because they appear to have simi- lar structural and biochemical properties. However, growing evidence suggests that HIF-1a and HIF-2a differentially contribute to tumor promotion. For example, the expression of HIF-1a and HIF-2a was different in various cancer cells ${ }^{[22]}$. Moreover, when HIF-1a or HIF-2a was overexpressed or silenced, different and sometimes opposite phenotypes were induced in various types of cancer cells ${ }^{[23-25]}$. To date, it is believed that cancer cells are addicted to one or both of the HIF-a proteins during transformation or growth. Therefore, the selective inhibition of HIF-1a or HIF-2a could be a better anticancer strategy than general HIF inhibition. Here, we found that HIF-2a is more effectively inhibited by disulfiram than HIF-1a in hepatoma cells. Accordingly, disulfiram could be used as an anticancer agent to selectively inhibit HIF-2aaddicted tumors.

With respect to cell survival, HIF-1a and HIF-2a exhibit opposite behavior. HIF-1a transactivates pro-apoptotic genes including BNIP3, but HIF-2a does not. In addition, HIF-1a induces cell cycle arrest under hypoxic conditions, whereas HIF-2a facilitates cell proliferation ${ }^{[26]}$. Mechanistically, HIF-1a 
antagonizes c-Myc and $\beta$-catenin by directly interfering with c-Myc/MAX and $\beta$-catenin/TCF dimerization ${ }^{[27,28]}$. HIF-2 $\alpha$ also interacts with c-Myc/MAX and $\beta$-catenin/TCF dimers but functionally boosts the transcriptional activities of these mitogenic factors ${ }^{[29,30]}$. Therefore, the selective inhibition of HIF-2a, rather than HIF-1 $\alpha$, may be more effective at inhibiting tumor growth because it targets hypoxic signaling and cell proliferation simultaneously. Following this concept, disulfiram may not only blunt tumor adaptation to hypoxia but also inhibit cell growth. This type of dual action of disulfiram was demonstrated in this study.

Previous studies on HIF-1a and HIF-2a regulation indicated that they share the same regulatory mechanism, PHDmediated prolyl hydroxylation, $\mathrm{pVHL}$-mediated ubiquitination, and $26 \mathrm{~S}$ proteasome-mediated proteolysis ${ }^{[31]}$. Therefore, it was surprising that HIF-1a and HIF-2a were differentially regulated by disulfiram. Some mechanism(s) other than the aforementioned process may be involved in the action of disulfiram. In fact, the stability of HIF-1a is known to be regulated by many proteins able to bind HIF-1a, whereas few studies have been performed to identify HIF-2a-interacting proteins $^{[32]}$. Therefore, it will be difficult to identify a disulfiram-responsive, HIF-2a-interacting protein. Currently, we can only speculate that HIF-2a-interacting proteins contain thiol-reactive domains, and thus, their binding properties are altered by disulfiram.

In the present study, we analyzed endothelial tube formation to examine the anti-angiogenic effect of disulfiram. This method is a type of bioassay that determines the amount of angiogenic molecules released into conditioned medium. Because VEGF is the major angiogenic factor released from cancer cells, tube formation is thought to be dependent on the level of VEGF in the conditioned medium. We demonstrated that disulfiram inhibited VEGF production in Hep3B cells and also that the conditioned media from disulfiram-pretreated cells failed to induce tube formation. Therefore, we propose that VEGF suppression by disulfiram results in impaired tube formation. However, we cannot rule out the possibility that disulfiram remaining in the conditioned media directly affected endothelial cells. The precise anti-angiogenic mechanism of disulfiram remains to be investigated.

In hypoxic cancer cells, HIF-a isoforms are regarded as the major transcription factors that drive the expression of VEGF. Because disulfiram deregulates HIF-2a expression during hypoxia, it was expected that disulfiram would block the hypoxic induction of VEGF. Indeed, we demonstrated that disulfiram reduced the level of VEGF mRNA in hypoxic Hep3B cells. In addition, disulfiram was found to reduce the secretion of VEGF protein from hypoxic Hep3B cells, which agrees with the above finding. However, it was surprising that disulfiram reduced the normoxic level of VEGF because HIF-a proteins were barely detected in Hep3B cells under normoxic conditions. To understand this unexpected finding, we re-measured the normoxic levels of the HIF-a proteins by enhancing their signals on Western blots. Of the HIF-a proteins, HIF-2a alone was detected, even under normoxia, and it was also suppressed by disulfiram. Indeed, many reports have demonstrated that HIF-2a, and not HIF-1a, was expressed and activated at near-physiological oxygen tensions ${ }^{[23]}$. In particular, HIF-2a is normoxically expressed in brain tumor cells, and it thus induces the pseudo-hypoxic phenotype in tumor behavior and angiogenesis ${ }^{[3]]}$. Therefore, it is plausible that a low level of HIF-2a maintains the basal expression of VEGF during normoxia. Therefore, disulfiram may reduce VEGF production in normoxic cells. Otherwise, disulfiram would inhibit other factors responsible for normoxic VEGF expression. VEGF expression is known to be regulated through multiple pathways. The VEGF promoter is targeted by other transcription factors, such as STAT3, NF-kB, AP-1/2, SP-1, Smads, estrogens, and prolactin, all of which can be activated under normoxia ${ }^{[34]}$. Which factor(s) is deregulated by disulfiram remains to be determined.

Disulfiram is not toxic if it is not taken with alcohol. The recommended dose of disulfiram ranges from $125 \mathrm{mg}$ to 500 $\mathrm{mg}$ daily, and the therapy is usually continued for months to years, depending on the patient's efforts to avoid drinking alcohol. Given that this drug has been used clinically for six decades, disulfiram may be a very safe drug. Therefore, if new usages of disulfiram are experimentally identified, disulfiram may be easily tested for new indications. However, before new clinical applications of disulfiram are implemented, it should be determined whether the effective doses of disulfiram are below its maximum permissible dose. In the present study, disulfiram was found to inhibit the expression of HIF-2 $\alpha$ and hypoxic genes at a concentration of 0.3-2.0 $\mathrm{mol} / \mathrm{L}$. Pharmacokinetic studies demonstrated that the disulfiram level in the plasma of patients is maintained over $0.5 \mu \mathrm{mol} / \mathrm{L}$ for $24 \mathrm{~h}$ after a single, oral administration of 250-mg disulfiram and that its peak level reaches $1.3 \mu \mathrm{mol} / \mathrm{L}$ at approximately $9 \mathrm{~h}$ after treatment ${ }^{[35]}$. Because the plasma levels of disulfiram are within the drug concentration range where its anti-HIF-2 effect was observed, we expect that the clinical application of disulfiram as a HIF-2-targeting anticancer drug will be realized in the future. However, more studies should be performed to better clarify the anti-HIF-2 action of disulfiram before its clinical application.

\section{Acknowledgements}

We thank Jae-hyun SONG, Burm-gi KIM, Han-young YOU, and Hyun-min $\mathrm{SO}$ for their contributions to this work. This work was supported by the Seoul Metropolitan Office of Education.

\section{Author contribution}

Hye-joon PARK, Min-sung KIM, and Chung-hyun CHO designed the research; Hye-joon PARK, Min-sung KIM, Kumsun $\mathrm{CHO}$, Jang-hyuk YUN, and Yong-joon CHOI performed research; Hye-joon PARK, Min-sung KIM, and Chung-hyun $\mathrm{CHO}$ analyzed data; and Hye-joon PARK and Chung-hyun $\mathrm{CHO}$ wrote the paper. 


\section{References}

1 Czyzyk-Krzeska MF. Molecular aspects of oxygen sensing in physiological adaptation to hypoxia. Respir Physiol 1997; 110: 99-111.

2 Wang GL, Semenza GL. Purification and characterization of hypoxiainducible factor 1. J Biol Chem 1995; 270: 1230-7.

3 Semenza GL. Regulation of mammalian $\mathrm{O}_{2}$ homeostasis by hypoxiainducible factor 1. Annu Rev Cell Dev Biol 1999; 15: 551-78.

4 Poon E, Harris AL, Ashcroft M. Targeting the hypoxia-inducible factor (HIF) pathway in cancer. Expert Rev Mol Med 2009; 11: e26.

5 Wright C, Moore RD. Disulfiram treatment of alcoholism. Am J Med 1990; 88: 647-55.

6 Tardito S, Bassanetti I, Bignardi C, Elviri L, Tegoni M, Mucchino C, et al. Copper binding agents acting as copper ionophores lead to caspase inhibition and paraptotic cell death in human cancer cells. J Am Chem Soc 2011; 133: 6235-42.

7 McDonnell NB, De Guzman RN, Rice WG, Turpin JA, Summers MF. Zinc ejection as a new rationale for the use of cystamine and related disulfidecontaining antiviral agents in the treatment of AIDS. J Med Chem 1997; 40: 1969-76.

8 Li L, Yang H, Chen D, Cui C, Dou QP. Disulfiram promotes the conversion of carcinogenic cadmium to a proteasome inhibitor with proapoptotic activity in human cancer cells. Toxicol Appl Pharmacol 2008; 229: 206-14.

9 Schirmer HK, Scott WW. Disulfiram and tumor inhibition. Trans Am Assoc Genitourin Surg 1966; 58: 63-6.

10 Wattenberg LW. Inhibition of dimethylhydrazine-induced neoplasia of the large intestine by disulfiram. J Natl Cancer Inst 1975; 54: 1005-6.

11 Marikovsky M, Nevo N, Vadai E, Harris-Cerruti C. Cu/Zn superoxide dismutase plays a role in angiogenesis. Int J Cancer 2002; 97: 34-41.

12 Wang W, McLeod HL, Cassidy J. Disulfiram-mediated inhibition of NFkappaB activity enhances cytotoxicity of 5-fluorouracil in human colorectal cancer cell lines. Int J Cancer 2003; 104: 504-11.

13 Shian SG, Kao YR, Wu FY, Wu CW. Inhibition of invasion and angiogenesis by zinc-chelating agent disulfiram. Mol Pharmacol 2003; 64: 1076-84.

14 Zhang H, Chen D, Ringler J, Chen W, Cui QC, Ethier SP, et al. Disulfiram treatment facilitates phosphoinositide 3-kinase inhibition in human breast cancer cells in vitro and in vivo. Cancer Res 2010; 70 : 3996-4004.

15 Loo TW, Clarke DM. Blockage of drug resistance in vitro by disulfiram, a drug used to treat alcoholism. J Natl Cancer Inst 2000; 92: 898902.

16 Chen D, Cui QC, Yang H, Dou QP. Disulfiram, a clinically used antialcoholism drug and copper-binding agent, induces apoptotic cell death in breast cancer cultures and xenografts via inhibition of the proteasome activity. Cancer Res 2006; 66: 10425-33.

17 Lin J, Haffner MC, Zhang Y, Lee BH, Brennen WN, Britton J, et al. Disulfiram is a DNA demethylating agent and inhibits prostate cancer cell growth. Prostate 2011; 71: 333-43.

18 Chun YS, Choi E, Kim GT, Choi H, Kim CH, Lee MJ, et al. Cadmium blocks hypoxia-inducible factor (HIF)-1-mediated response to hypoxia by stimulating the proteasome-dependent degradation of HIF-1alpha. Eur J Biochem 2000; 267: 4198-204.

19 Karila L, Reynaud M, Aubin HJ, Rolland B, Guardia D, Cottencin O, et al. Pharmacological treatments for cocaine dependence: is there something new? Curr Pharm Des 2011; 17: 1359-68.

20 Veien NK. Systemic contact dermatitis. Int J Dermatol 2011; 50: $1445-56$.

21 Kanai K, Ito Y, Nagai N, Itoh N, Hori Y, Chikazawa S, et al. Effects of instillation of eyedrops containing disulfiram and hydroxypropyl- $\beta$ cyclodextrin inclusion complex on endotoxin-induced uveitis in rats. Curr Eye Res 2012; 37: 124-31.

22 Sowter HM, Raval RR, Moore JW, Ratcliffe PJ, Harris AL. Predominant role of hypoxia-inducible transcription factor (Hif)-1alpha versus Hif-2alpha in regulation of the transcriptional response to hypoxia. Cancer Res 2003; 63: 6130-4.

23 Löfstedt T, Fredlund E, Holmquist-Mengelbier L, Pietras A, Ovenberger $\mathrm{M}$, Poellinger $\mathrm{L}$, et al. Hypoxia inducible factor-2alpha in cancer. Cell Cycle 2007; 6: 919-26.

24 Imamura T, Kikuchi H, Herraiz MT, Park DY, Mizukami Y, Mino-Kenduson $\mathrm{M}$, et al. HIF-1alpha and HIF-2alpha have divergent roles in colon cancer. Int J Cancer 2009; 124: 763-71.

25 Beasley NJ, Leek R, Alam M, Turley H, Cox GJ, Gatter K, et al. Hypoxiainducible factors HIF-1alpha and HIF-2alpha in head and neck cancer: relationship to tumor biology and treatment outcome in surgically resected patients. Cancer Res 2002; 62: 2493-7.

26 Loboda A, Jozkowicz A, Dulak J. HIF-1 and HIF-2 transcription factors - similar but not identical. Mol Cells 2010; 29: 435-42.

27 Koshiji M, Kageyama Y, Pete EA, Horikawa I, Barrett JC, Huang LE. HIF-1alpha induces cell cycle arrest by functionally counteracting Myc. EMBO J 2004; 23: 1949-56.

28 Kaidi A, Williams AC, Paraskeva C. Interaction between beta-catenin and HIF-1 promotes cellular adaptation to hypoxia. Nat Cell Biol 2007; 9: 210-7.

29 Gordan JD, Bertout JA, Hu CJ, Diehl JA, Simon MC. HIF-2alpha promotes hypoxic cell proliferation by enhancing c-myc transcriptional activity. Cancer Cell 2007; 11: 335-47.

30 Choi H, Chun YS, Kim TY, Park JW. HIF-2alpha enhances betacatenin/TCF-driven transcription by interacting with beta-catenin. Cancer Res 2010; 70: 10101-11.

31 Chun YS, Kim MS, Park JW. Oxygen-dependent and -independent regulation of HIF-1alpha. J Korean Med Sci 2002; 17: 581-8.

32 Semenza GL. Hypoxia-inducible factor 1 (HIF-1) pathway. Sci STKE 2007; 407: cm8.

33 Pietras A, Johnsson AS, PåhIman S. The HIF-2 $\alpha$-driven pseudo-hypoxic phenotype in tumor aggressiveness, differentiation, and vascularization. Curr Top Microbiol Immunol 2010; 345: 1-20.

34 Birk DM, Barbato J, Mureebe L, Chaer RA. Current insights on the biology and clinical aspects of VEGF regulation. Vasc Endovascular Surg 2008; 42: 517-30.

35 Faiman MD, Jensen JC, Lacoursiere RB. Elimination kinetics of disulfiram in alcoholics after single and repeated doses. Clin Pharmacol Ther 1984; 36: 520-6. 